\title{
BMR
}

\section{Quantitative trait locus analysis of leaf morphological characters, yield-related traits, and secondary metabolite contents in Eucommia ulmoides}

\author{
Y. Li, J.K. Wei, Z.Q. Li, A.N. Lei and M.H. Liu \\ College of Forestry, Northwest A\&F University, Yangling, Shaanxi, China \\ Corresponding author: Z.Q. Li \\ E-mail: zqlinwafu@163.com / lizhouqi@nwsuaf.edu.cn
}

Genet. Mol. Res. 14 (4): 17871-17884 (2015)

Received August 26, 2015

Accepted October 11, 2015

Published December 22, 2015

DOI http://dx.doi.org/10.4238/2015.December.22.12

\begin{abstract}
Eucommia ulmoides is cultivated for the production of eucommia rubber and Chinese herbal drugs. Molecular breeding methods, such as marker-assisted selection (MAS), have the potential to improve the efficiency of E. ulmoides breeding. Quantitative trait locus (QTL) analysis was applied to identify marker-trait associations for $E$. ulmoides using an $F_{1}$ mapping population of 152 individuals derived from a cross between the wild genotype Xiaoye and the cultivar Qinzhong No. 1. A total of 78 QTLs were identified for 12 leaf traits involving morphology, yield, and secondary metabolites. Phenotypic variance explained by individual QTLs ranged from 10.4 to $53.3 \%$. Fifteen QTL clusters, each harboring loci controlling at least two leaf traits, were detected across nine linkage groups. Co-location of these QTLs may be due to pleiotropy or linkage. Three main QTL regions for secondary metabolites were consistently identified each year. QTL information from this study furthers our understanding of the genetic architecture of these economically important traits and of MAS in E. ulmoides breeding.
\end{abstract}

Key words: Eucommia ulmoides; Leaf morphology; Secondary metabolite; Yield; Quatitative trait locus 


\section{INTRODUCTION}

Rubber is an important raw material used in the chemical industry. Natural rubber produced from Brazilian rubber trees consists entirely of cis-polyisoprene. Eucommia ulmoides Oliver $(2 n=34)$, the only species of the genus Eucommia in the family Eucommiaceae, produces trans-polyisoprene, which is distinguished as eucommia rubber (EU-rubber) (Hu, 1979). EU-rubber is obtained from the whole tree except for the xylem, and is a hard rubber with thermoplasticity and properties similar to those of plastic (Nakazawa et al., 2009). In China, Japan, and Russia, E. ulmoides is cultivated for EU-rubber, which is used as an insulator in the manufacture of wire rope, submarine cables, and dental supplies.

E. ulmoides is also an economically important plant for traditional Chinese medicine. The main bioactive constituents in this plant are phenylpropanoids (e.g., chlorogenic acid) and flavonoids (e.g., rutin). These secondary metabolites are found in the bark and leaves and possess many pharmacological activities, which are anti-hypertensive (Kwan et al., 2003), anti-hyperglycemic (Lee et al., 2005), anti-oxidant (Hsieh and Yen, 2000), and anti-mutagenic (Nakamura et al., 1997). In addition, foods prepared from the bark, leaves, and flowers have health care functions, which involve strengthening the body, enhancing metabolism, and combating fatigue.

Historically, only the bark of E. ulmoides was officially recognized as a traditional Chinese herbal drug. In the 1980s, uncontrolled harvesting for the bark almost resulted in the loss of this wild resource. Recent research has confirmed that the chemical constituents of the leaves are similar to those of the bark (Takamura et al., 2007). Together with the bark, the leaves have also been listed in "Chinese Pharmacopoeia" since 2005 (China Pharmacopoeia Committee, 2005). Secondary metabolites in E. ulmoides are now obtained mainly from the leaves. Therefore, the exploitation and utilization of leaves is very promising for the protection of this plant resource and for preventing the loss of E. ulmoides.

To improve the yield and quality of the leaves, E. ulmoides breeding programs have traditionally been conducted by selecting promising trees from wild populations. These selected trees have then been subjected to vegetative propagation and released as clones (Li et al., 2014a). Nowadays, breeders have introduced controlled crosses between divergent clones to achieve larger gains per unit effort (Li et al., 2014b). However, the selection of cultivars using conventional phenotypic evaluation is a time-consuming and labor-intensive activity.

The use of molecular markers for indirect selection of phenotypic traits, or markerassisted selection (MAS), could improve the efficiency of traditional breeding. The success of MAS depends on the identification of reliable molecular markers linked to economically important traits, and quantitative trait locus (QTL) analysis can be used to identify markertrait associations. The genetic information acquired by QTL analysis, including the number, location, and magnitude of effects of genetic regions associated with a trait, also contributes to our understanding of the genetic basis of economically important traits. In tree species, QTL analyses have been reported for leaf traits and chemical constituents in European beech (Scalfi et al., 2004), oak (Gailing et al., 2013), Eucalyptus (Freeman et al., 2009), peach (Eduardo et al., 2013), and oil palm (Montoya et al., 2013). In contrast, QTLs affecting leaf morphology characters, yield-related traits, and secondary metabolite contents have not been reported in E. ulmoides. 
Recently, we constructed a genetic linkage map of $E$. ulmoides using an $\mathrm{F}_{1}$ mapping population (DZ0901) derived from a cross between the wild genotype Xiaoye and the cultivar Qinzhong No.1 ( $\mathrm{Li}$ et al., 2014c). The female parent, Xiaoye, has small leaves and low levels of secondary metabolites, whereas the male parent, Qinzhong No.1, has large leaves and high levels of secondary metabolites. The genetic linkage map contains a total of 706 molecular markers, including 515 sequence-related amplified polymorphism markers, 117 amplified fragment length polymorphism markers, 18 inter-simple sequence repeat markers, and 56 simple sequence repeat markers. The total map distance was $2133 \mathrm{cM}$, and the average map distance between adjacent markers was $3.1 \mathrm{cM}$. The genetic linkage map was used to identify QTLs that affect growthrelated traits. In this report, we describe QTL analysis of 12 leaf traits involving morphology, yield, and secondary metabolites. The objective of our investigation was to acquire information on the association between molecular markers and economically important traits in E. ulmoides and to improve our understanding of the inheritance of these traits, which can then be used to facilitate MAS in breeding programs.

\section{MATERIAL AND METHODS}

\section{Plant materials}

The DZ0901 mapping population consisted of $152 \mathrm{~F}_{1}$ individuals derived from a cross between the wild genotype Xiaoye and the cultivar Qinzhong No.1. The parents differ in leaf morphology characters and in secondary metabolite content, and originated from two areas with different climatic and geographic conditions (Li et al., 2014c). Seedlings were grown in a greenhouse in 2010 and transferred to a field at the nursery of Northwest A\&F University in March 2011.

\section{Phenotypic measurements}

Three healthy leaves were collected from each progeny plant in mid-September 2011 and 2012. Leaf morphology characters and single leaf weight were measured, and mean values were used for further analysis. The six leaf morphological characteristics were leaf area, leaf length, leaf width, leaf length-width ratio, petiole length, and number of veins. Leaf area, leaf length, and leaf width were determined using a leaf area meter (Beijing Yaxinliyi Science and Technology Co. Ltd., Beijing, China). For the yield traits, single leaf weight was determined after drying leaves at $105^{\circ} \mathrm{C}$ for $90 \mathrm{~min}$, and total dry weight was estimated by multiplying the single leaf weight by the number of leaves of each plant.

For each plant, two leaf samples (10-15 random leaves per sample) were collected to determine the levels of chlorogenic acid, rutin, and EU-rubber; results are reported as mean values. The green leaves were dried at $105^{\circ} \mathrm{C}$ for $90 \mathrm{~min}$ and pulverized using a disintegrator (FW-200, Zhongxingweiye Instrument Co. Ltd., Beijing, China). The contents of chlorogenic acid and rutin were determined using high-performance liquid chromatography (HPLC) analysis. A sample of $500 \mathrm{mg}$ leaf meal was weighed accurately into a conical flask with a plug, $20 \mathrm{~mL}$ $60 \%$ ethanol was added, and the leaf meal was dissolved for $60 \mathrm{~min}$. Next, the flask was set 
to a KQ-600DV ultrasonic wave generator (Kunshan Ultrasonic Equipment Co. Ltd., Shanghai, China) for extraction at $40^{\circ} \mathrm{C}$ for $30 \mathrm{~min}$. The extract was filtered and the residue was extracted again using $10 \mathrm{~mL} 60 \%$ ethanol for a further $30 \mathrm{~min}$. The obtained filtrate was combined and diluted with $60 \%$ ethanol in a $50-\mathrm{mL}$ volumetric flask. The solution was filtered through a $0.45-$ $\mu \mathrm{m}$ microporous filtering film. The resulting filtrate was used to measure the levels of chlorogenic acid and rutin by HPLC (L2000, Hitachi Ltd., Tokyo, Japan). Separation was carried out in a C18 column (Agilent Zorbax SB, 4.6 × 250 mm, $5 \mu \mathrm{m}$, Agilent, Santa Clara, CA, USA). The mobile phase was methanol-water-acetic acid (50:49:1, v/v) with a flow rate of $1 \mathrm{~mL} / \mathrm{min}$. The injection amount was $10 \mu \mathrm{L}$, the detection wave length was $340 \mathrm{~nm}$, and the column temperature was $32^{\circ} \mathrm{C}$. The contents of chlorogenic acid and rutin were calculated using standard curves based on the corresponding peak areas in HPLC. To determine the EU-rubber content, the protocol reported by Ma et al. (1994) was adapted. Briefly, $3.0 \mathrm{~g}$ leaf meal was weighed accurately into a conical flask and treated in the same way as described above using $60 \%$ ethanol. The obtained residue was transferred to another conical flask, $100 \mathrm{~mL} 10 \% \mathrm{NaOH}$ was added, and the residue was soaked at $90^{\circ} \mathrm{C}$ for $3 \mathrm{~h}$. The $\mathrm{NaOH}$-soluble material was removed, and the residue was extracted in $100 \mathrm{~mL} 10 \% \mathrm{NaOH}$ at $90^{\circ} \mathrm{C}$ for a further $3 \mathrm{~h}$. The crude extract was treated in concentrated hydrochloric acid at $40^{\circ} \mathrm{C}$ for $2 \mathrm{~h}$. The obtained EU-rubber was air-dried and weighed. The content was calculated by dividing the weight of EU-rubber by the weight of leaf meal.

\section{QTL mapping}

Analyses of means, standard errors, and frequency distributions as well as correlations involving 12 measured traits were carried out using the analysis tools of SPSS 13.0 (SPSS Inc., Chicago, IL, USA, 2004). QTL analysis was conducted using the genetic linkage map constructed in a previous study (Li et al., 2014c). MapQTL 5.0 was used for this analysis (Van Ooijen, 2004). First, the nonparametric Kruskal-Wallis test was performed to individually associate single markers and traits. Next, interval mapping analyses were performed to locate preliminary QTL positions on the map. Finally, multiple-QTL mapping was undertaken, and the markers closest to the QTL peaks detected by interval mapping were used as cofactors. The logarithm of odds (LOD) thresholds were estimated with a 1000-permutation test. QTLs with LOD values higher than the genome-wide threshold at $\mathrm{P}<0.05$ were considered significant. However, those QTLs with an LOD score $\geq 3$ and smaller than the threshold were also reported. QTL positions in the genetic linkage map were drawn using MapChart 2.2 (Voorrips, 2002).

\section{RESULTS}

\section{Phenotypic variability and correlation}

Considerable variation was found in leaf morphology characters, yield-related traits, and levels of secondary metabolites in the DZ0901 mapping population (Table 1). The coefficients of variation of leaf area, single leaf weight, number of leaves, total dry weight, chlorogenic acid, and rutin were high, varying from $30.1 \%$ (number of leaves) to $45.1 \%$ (total dry weight), and from $33.3 \%$ (single leaf weight) to $60.9 \%$ (total dry weight) in 2011 and 2012, respectively. The coefficients of variation of other traits were medium, varying from $8.8 \%$ (number of veins) to $19.0 \%$ (petiole length) and from $9.9 \%$ (number of veins) to 24.6\% (EU-rubber) in 2011 and 2012, respectively. Frequency distributions of these traits are presented in Figure 1. 
Pairwise correlation coefficients among the 12 phenotypic traits are presented in Table 2. Highly significant positive correlations were found between several morphological characters or yield-related traits in both years. Associations were found for leaf area, leaf length, leaf width, petiole length, number of veins, single leaf weight, and total dry weight. The highest correlations found were between leaf area and leaf length $(r=0.91,0.88)$, leaf area and leaf width $(r=0.95$, $0.96)$, leaf area and single leaf weight $(r=0.76,0.89)$, leaf length and leaf width $(r=0.84,0.75)$, leaf length and single leaf weight $(r=0.70,0.79)$, leaf width and single leaf weight $(r=0.74,0.84)$, and single leaf weight and total dry weight $(r=0.78,0.75)$, in 2011 and 2012, respectively. The two secondary metabolites, chlorogenic acid and rutin, were found to be positively correlated with each other. Correlation coefficients between secondary metabolites and other leaf traits were relatively low. Significant positive correlations between years were found for leaf width, leaf length-width ratio, petiole length, single leaf weight, number of leaves, total dry weight, chlorogenic acid, and rutin; however, Pearson's correlation coefficients were generally low indicating that there was a strong ontogenic and/or environmental effect on leaf development.

Table 1. Mean, standard deviation (SD), range, and coefficient of variation (CV) for the leaf traits.

\begin{tabular}{|c|c|c|c|c|c|}
\hline Traits & Mean & SD & Minimum & Maximum & $\mathrm{CV}(\%)$ \\
\hline Leaf area $2011\left(\mathrm{~mm}^{2}\right)$ & 8851.4 & 2786.2 & 2244.2 & 16505.2 & 31.5 \\
\hline Leaf area $2012\left(\mathrm{~mm}^{2}\right)$ & 5262.1 & 1799.2 & 2114.6 & 11506.5 & 34.2 \\
\hline Leaf length $2011(\mathrm{~mm})$ & 153.6 & 26.3 & 71.0 & 213.3 & 17.1 \\
\hline Leaf length $2012(\mathrm{~mm})$ & 131.6 & 21.7 & 83.0 & 191.0 & 16.5 \\
\hline Leaf width 2011 (mm) & 75.4 & 12.7 & 40.3 & 104.3 & 16.8 \\
\hline Leaf width 2012 (mm) & 59.8 & 11.1 & 37.1 & 93.4 & 18.6 \\
\hline Leaf length-width ratio 2011 & 2.0 & 0.2 & 1.5 & 2.6 & 10.0 \\
\hline Leaf length-width ratio 2012 & 2.2 & 0.3 & 1.5 & 3.2 & 13.6 \\
\hline Petiole length 2011 (mm) & 17.4 & 3.3 & 10.7 & 27.1 & 19.0 \\
\hline Petiole length $2012(\mathrm{~mm})$ & 15.0 & 3.0 & 7.6 & 23.6 & 20.0 \\
\hline Number of veins 2011 & 13.6 & 1.2 & 10.3 & 17.0 & 8.8 \\
\hline Number of veins 2012 & 16.1 & 1.6 & 11.7 & 21.0 & 9.9 \\
\hline Single leaf weight 2011 (g) & 2.2 & 0.7 & 0.5 & 4.1 & 31.8 \\
\hline Single leaf weight $2012(\mathrm{~g})$ & 0.6 & 0.2 & 0.2 & 1.4 & 33.3 \\
\hline Number of leaves 2011 & 29.6 & 8.9 & 11.0 & 55.0 & 30.1 \\
\hline Number of leaves 2012 & 114.3 & 45.0 & 43.0 & 267.3 & 39.4 \\
\hline Total dry weight 2011 (g) & 65.4 & 29.5 & 9.3 & 157.1 & 45.1 \\
\hline Total dry weight $2012(\mathrm{~g})$ & 65.7 & 40.0 & 12.0 & 222.1 & 60.9 \\
\hline Chlorogenic acid 2011 (\%) & 4.5 & 1.5 & 0.4 & 7.4 & 33.3 \\
\hline Chlorogenic acid 2012 (\%) & 2.5 & 1.2 & 0.4 & 5.5 & 48.0 \\
\hline Rutin 2011 (\%) & 0.5 & 0.2 & 0.1 & 1.2 & 40.0 \\
\hline Rutin 2012 (\%) & 0.5 & 0.2 & 0.2 & 1.3 & 40.0 \\
\hline Eucommia rubber 2011 (\%) & 4.5 & 0.6 & 2.9 & 6.5 & 13.3 \\
\hline Eucommia rubber $2012(\%)$ & 5.7 & 1.4 & 2.4 & 10.4 & 24.6 \\
\hline
\end{tabular}

\section{QTL analysis}

A total of 78 QTLs were identified for the 12 leaf traits (Figure 2, Table 3). Phenotypic variance explained by individual QTL ranged from $10.4 \%$ for Dca2-5 to $53.3 \%$ for Dca2-6. Of these QTLs, 38 were significant at a genome level, and others had a LOD score $\geq 3$. Results obtained from the Kruskal-Wallis nonparametric test revealed that 44 flanking markers nearest to the QTL position were significantly associated with these traits. QTLs detected in both years with overlapping confidence intervals were mapped for single leaf weight on LG22 (Ddlw1-1 and Ddlw24), for chlorogenic acid on LG1 (Dca1-2 and Dca2-2), for rutin on LG9 (Dru1-3 and Dru2-5), and for EU-rubber on LG1 (Deur1-1 and Deur2-1). 

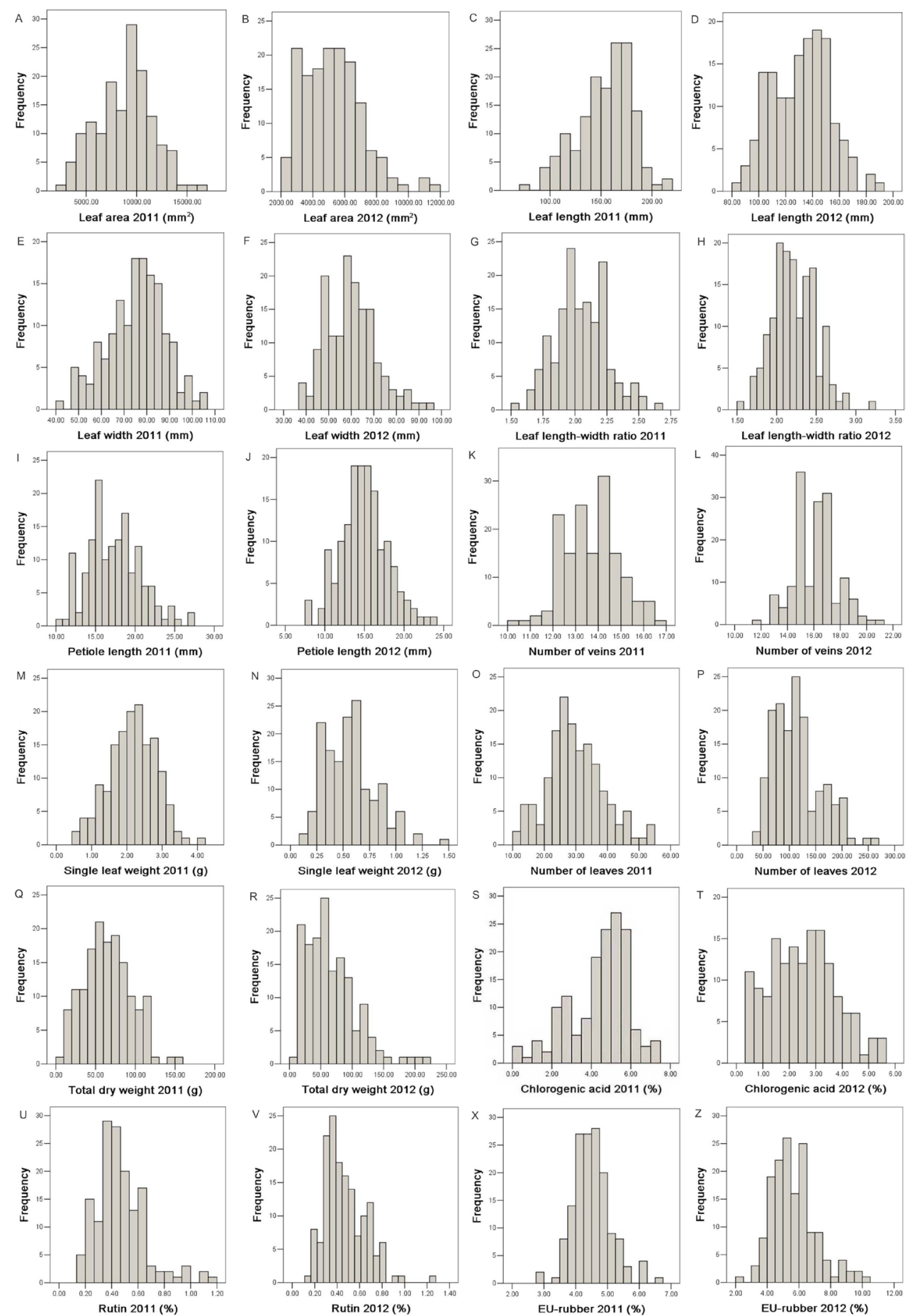

Figure 1. Frequency distribution of leaf traits for the DZ0901 population. 
Table 2. Pearson correlation coefficients between the leaf traits.

\begin{tabular}{|c|c|c|c|c|c|c|c|c|c|c|c|c|}
\hline Traits & LA & LL & LW & LWR & PL & NV & SLW & $\mathrm{NL}$ & TDW & $\mathrm{CA}$ & $\mathrm{RU}$ & EUR \\
\hline LA & 0.11 & $0.91^{* *}$ & $0.95^{\star *}$ & -0.01 & $0.67^{\star \star}$ & $0.42^{\star *}$ & $0.76^{* *}$ & 0.07 & $0.51^{\star *}$ & 0.07 & $-0.18^{*}$ & 0.13 \\
\hline LL & $0.88^{* *}$ & -0.00 & $0.84^{\star *}$ & $0.34^{\star *}$ & $0.61^{\text {** }}$ & $0.43^{* *}$ & $0.70^{\star \star}$ & 0.08 & $0.49^{* *}$ & 0.14 & -0.08 & $0.19^{*}$ \\
\hline LW & $0.96^{\star *}$ & $0.75^{\star \star}$ & $0.18^{*}$ & $-0.23^{\star *}$ & $0.67^{\star *}$ & $0.42^{\star \star}$ & $0.74^{\star *}$ & 0.09 & $0.52^{\star \star}$ & 0.10 & -0.15 & 0.12 \\
\hline LWR & $-0.23^{\star \star}$ & $0.22^{* \star}$ & $-0.47^{\star *}$ & $0.26^{* *}$ & -0.07 & 0.05 & -0.03 & 0.01 & -0.02 & 0.07 & 0.09 & 0.11 \\
\hline PL & $0.65^{* *}$ & $0.57^{\star *}$ & $0.62^{\star *}$ & -0.12 & $0.34^{* *}$ & $0.37^{\star *}$ & $0.60^{\star *}$ & $0.26^{\star *}$ & $0.55^{\star *}$ & $-0.18^{*}$ & $-0.22^{\star \star}$ & 0.04 \\
\hline NV & $0.57^{\text {** }}$ & $0.66^{\star *}$ & $0.49^{\star *}$ & 0.13 & $0.27^{\text {** }}$ & 0.13 & $0.43^{\star *}$ & 0.04 & $0.30^{* *}$ & -0.05 & -0.06 & $0.24^{* *}$ \\
\hline SLW & $0.89^{* *}$ & $0.79^{* *}$ & $0.84^{\star *}$ & $-0.19^{*}$ & $0.60^{* *}$ & $0.57^{\text {** }}$ & $0.22^{\star \star}$ & $0.24^{\star *}$ & $0.78^{\text {** }}$ & -0.03 & -0.13 & $0.18^{*}$ \\
\hline NL & 0.02 & -0.05 & 0.06 & $-0.17^{*}$ & 0.11 & -0.02 & 0.12 & $0.50^{\star *}$ & $0.76^{\star *}$ & $-0.19^{*}$ & 0.06 & 0.02 \\
\hline TDW & $0.63^{* *}$ & $0.52^{\star *}$ & $0.61^{\star *}$ & $-0.21^{\text {** }}$ & $0.49^{* *}$ & $0.38^{\text {** }}$ & $0.75^{\star \star}$ & $0.69^{\star *}$ & $0.47^{\text {** }}$ & -0.13 & -0.02 & 0.15 \\
\hline CA & 0.10 & 0.07 & 0.03 & 0.05 & 0.07 & $0.16^{*}$ & 0.10 & -0.04 & 0.07 & $0.31^{\text {** }}$ & $0.35^{\star \star}$ & -0.05 \\
\hline RU & 0.02 & -0.09 & 0.06 & $-0.23^{* *}$ & 0.01 & 0.01 & 0.06 & $0.22^{* *}$ & $0.19^{*}$ & $0.54^{\star *}$ & $0.35^{\star *}$ & -0.03 \\
\hline EUR & -0.07 & -0.05 & -0.03 & -0.06 & -0.03 & -0.07 & -0.04 & 0.05 & 0.00 & $-0.20^{*}$ & 0.02 & 0.11 \\
\hline
\end{tabular}

Above diagonal: correlation coefficients for the traits measured in 2011, below diagonal: correlation coefficients for the traits measured in 2012, diagonal: between-year correlation coefficients (in italics); ${ }^{*} \mathrm{P} \leq 0.05,{ }^{* *} \mathrm{P} \leq 0.01$; LA: leaf area, LL: leaf length, LW: leaf width, LWR: leaf length-width ratio, PL: petiole length, NV: number of veins, SLW: single leaf weight, NL: number of leaves, TDW: total dry weight, CA: content of chlorogenic acid, RU: content of rutin, EUR: content of eucommia rubber.

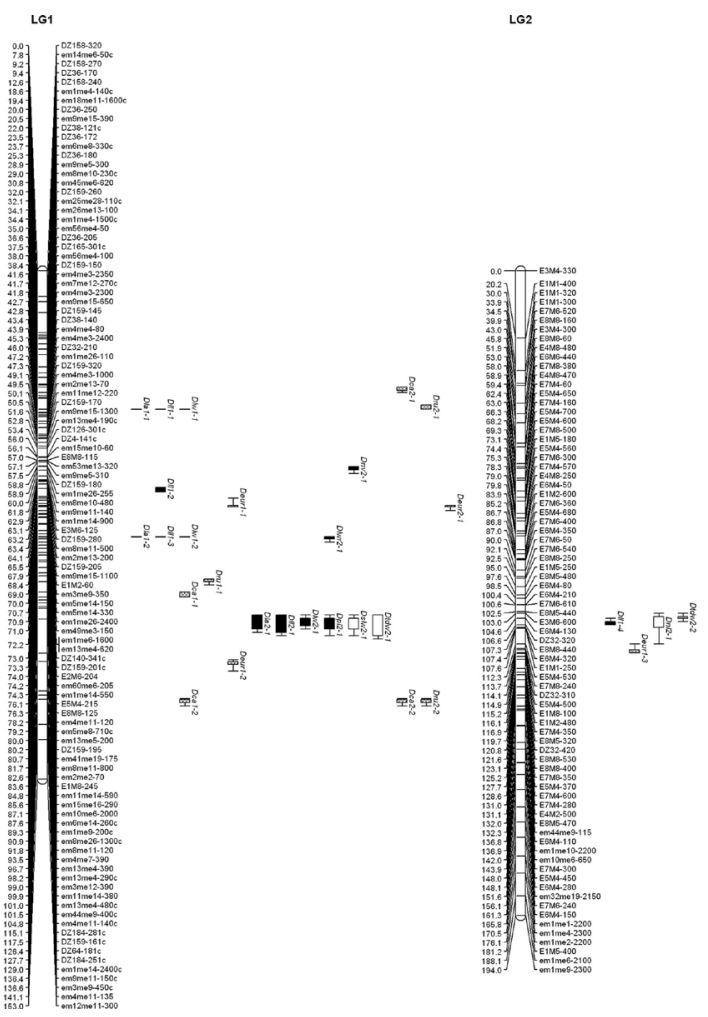

Figure 2. Locations of quantitative trait loci (QTLs) for leaf traits on the linkage groups (LG) of Eucommia ulmoides from the DZ0901 mapping population. The names of markers are given on the right side of the LGs. The positions (in $\mathrm{CM}$ ) of markers are indicated on the left side of each LG. QTLs are represented by vertical bars positioned to the right of each LG. Thin lines correspond to LOD-2 and thick bars to LOD-1 confidence intervals. Solid bars represent QTLs for leaf morphological characters. Blank bars represent QTLs for yield-related traits. Bars filled with two-sided oblique lines represent QTLs for secondary metabolite contents. The names of the QTLs are presented in Table 3.

Continued on next page 
Figure 2. Continued.
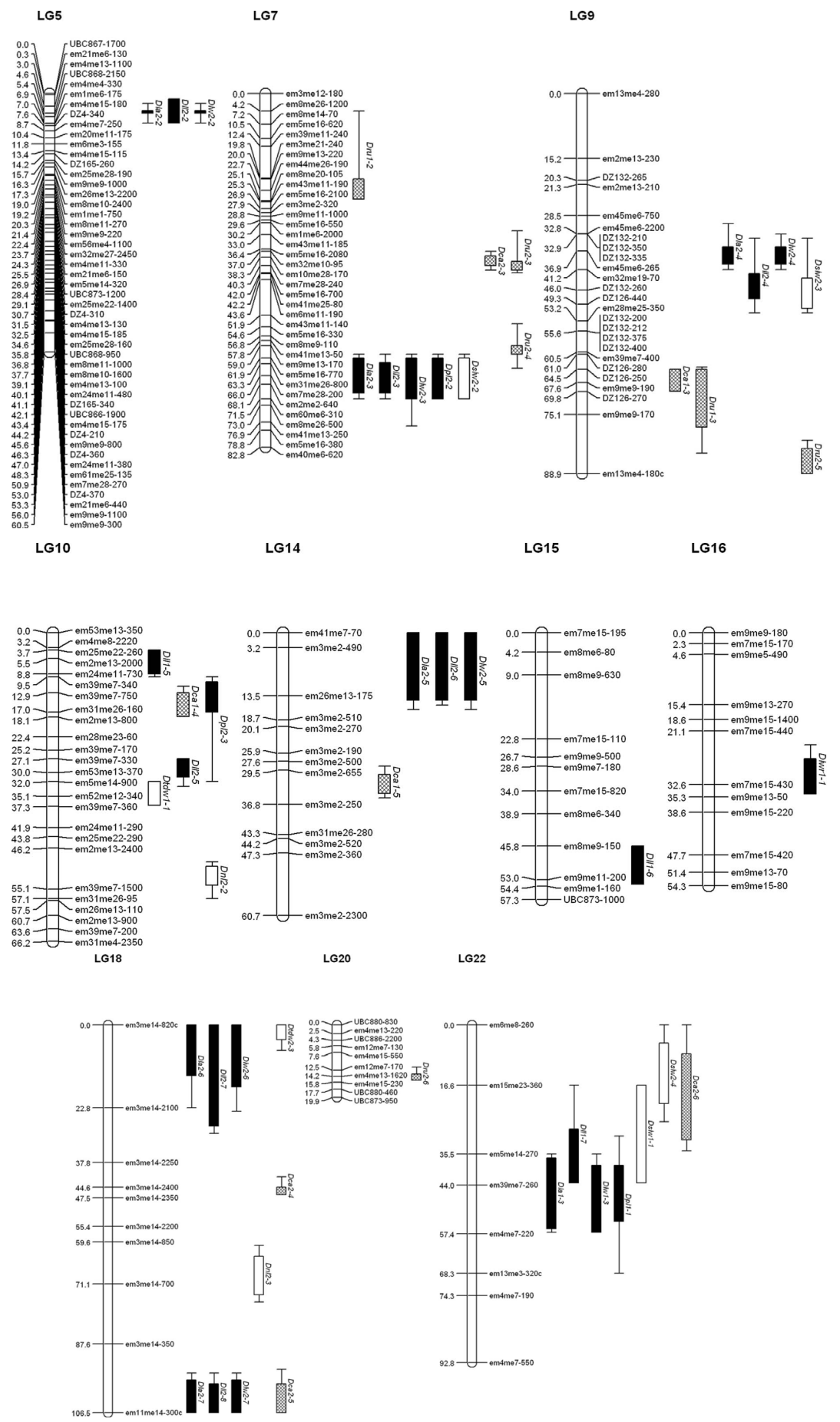
Table 3. QTLs for leaf traits detected in the DZ0901 population.

\begin{tabular}{|c|c|c|c|c|c|c|c|}
\hline Traits $^{a}$ & $\mathrm{QTL}^{b}$ & Linkage group & Position $(\mathrm{cM})^{c}$ & $\mathrm{LOD}^{d}$ & Marker ${ }^{e}$ & $\%$ Var. $^{f}$ & $\mathrm{KW}^{g}$ \\
\hline \multirow[t]{3}{*}{ LA 2011} & Dla1-1 & LG1 & 41.8 & $4.6^{\star \star}$ & em4me3-2300 & 16.7 & - \\
\hline & Dla1-2 & LG1 & 80.0 & $5.3^{* *}$ & em13me5-200 & 14.9 & 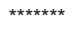 \\
\hline & Dla1-3 & LG22 & 42.5 & 3.5 & em39me7-260 & 31.2 & - \\
\hline \multirow[t]{7}{*}{ LA 2012} & Dla2-1 & LG1 & 104.5 & $7.2^{\text {** }}$ & em4me11-140c & 23.2 & $\star \star * * * * * *$ \\
\hline & Dla2-2 & LG5 & 4.6 & $4.4^{\star *}$ & UBC868-2150 & 34.2 & - \\
\hline & Dla2-3 & LG7 & 62.9 & $5.7^{\star \star}$ & em31me26-800 & 19.0 & $\star \star \star * \star \star * *$ \\
\hline & Dla2-4 & LG9 & 37.9 & 3.4 & em45me6-265 & 18.7 & $* *$ \\
\hline & Dla2-5 & LG14 & 8.2 & 3.8 & em3me2-490 & 13.9 & $\star \star \star \star *$ \\
\hline & Dla2-6 & LG18 & 3.0 & $4.6^{\star \star}$ & em3me14-820c & 28.9 & $\star *$ \\
\hline & Dla2-7 & LG18 & 103.6 & 3.2 & em11me14-300c & 13.3 & $\star \star \star \star *$ \\
\hline \multirow[t]{7}{*}{ LL 2011} & DII1-1 & LG1 & 41.7 & $5.1^{\text {** }}$ & em7me12-270c & 14.4 & $\star \star \star * \star * \star *$ \\
\hline & DII1-2 & LG1 & 65.1 & $5.7^{\star \star}$ & DZ159-205 & 27.9 & - \\
\hline & DII1-3 & LG1 & 80.0 & $4.7^{\star \star}$ & em13me5-200 & 13.9 & $* \star * * * \star * *$ \\
\hline & DII1-4 & LG2 & 106.7 & 3.2 & DZ32-320 & 26.8 & $* \star *$ \\
\hline & DI/1-5 & LG10 & 8.8 & 3.0 & em24me11-730 & 13.2 & - \\
\hline & DII1-6 & LG15 & 50.8 & 3.1 & em9me11-200 & 48.4 & - \\
\hline & DII1-7 & LG22 & 39.5 & 3.3 & em5me14-270 & 31.2 & - \\
\hline \multirow[t]{8}{*}{ LL 2012} & DII2-1 & LG1 & 107.8 & $6.6^{\star *}$ & em4me11-140c & 31.9 & $\star \star \star \star * \star * *$ \\
\hline & DII2-2 & LG5 & 2.2 & 4.2 & em4me13-1100 & 33.6 & ** \\
\hline & DII2-3 & LG7 & 67.0 & $6.3^{* *}$ & em7me28-200 & 28.0 & $\star \star \star * \star * \star * *$ \\
\hline & DI/2-4 & LG9 & 45.2 & 3.9 & DZ132-260 & 21.7 & - \\
\hline & DI/2-5 & LG10 & 29.1 & $4.5^{\star \star}$ & em53me13-370 & 41.3 & - \\
\hline & DII2-6 & LG14 & 9.2 & $4.6^{\text {** }}$ & em26me13-175 & 18.1 & - \\
\hline & DII2-7 & LG18 & 1.0 & $4.3^{\star *}$ & em3me14-820c & 24.8 & - \\
\hline & DI/2-8 & LG18 & 104.6 & 3.5 & em11me14-300c & 12.3 & $* \star * *$ \\
\hline \multirow[t]{3}{*}{ LW 2011} & $D / w 1-1$ & LG1 & 41.8 & $5.0^{* *}$ & em4me3-2300 & 18.6 & - \\
\hline & $D / w 1-2$ & LG1 & 80.0 & $5.1^{\text {** }}$ & em13me5-200 & 14.4 & $* * * * * * * *$ \\
\hline & D/w1-3 & LG22 & 44.0 & 3.8 & em39me7-260 & 32.4 & - \\
\hline \multirow[t]{7}{*}{ LW 2012} & D/w2-1 & LG1 & 104.5 & $8.4^{\star \star}$ & em4me11-140c & 26.4 & $\star \star \star * \star * \star *$ \\
\hline & D/w2-2 & LG5 & 4.6 & 3.4 & UBC868-2150 & 28.4 & - \\
\hline & D/w2-3 & LG7 & 68.0 & 4.2 & em2me2-640 & 16.4 & 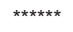 \\
\hline & $D / w 2-4$ & LG9 & 37.9 & 3.0 & em45me6-265 & 17.9 & - \\
\hline & D/w2-5 & LG14 & 5.2 & 3.7 & em3me2-490 & 12.1 & $\star \star \star \star *$ \\
\hline & D/w2-6 & LG18 & 5.0 & $4.4^{\star \star}$ & em3me14-820c & 28.4 & $* *$ \\
\hline & DIw2-7 & LG18 & 103.6 & 3.5 & em11me14-300c & 14.8 & $\star \star \star * *$ \\
\hline LWR 2011 & Dlwr1-1 & LG16 & 31.1 & 3.4 & em7me15-430 & 41.4 & - \\
\hline LWR 2012 & D/wr2-1 & LG1 & 80.0 & 3.2 & em13me5-200 & 15.7 & * \\
\hline PL 2011 & $D p / 1-1$ & LG22 & 42.5 & 3.6 & em39me7-260 & 33.9 & - \\
\hline \multirow[t]{3}{*}{ PL 2012} & $D p / 2-1$ & LG1 & 105.8 & $5.5^{\star \star}$ & em4me11-140c & 19.8 & $* * * * * * *$ \\
\hline & $D p / 2-2$ & LG7 & 70.1 & $4.9^{\star *}$ & em60me6-310 & 18.5 & $* * * * * * *$ \\
\hline & Dp/2-3 & LG10 & 14.9 & 3.3 & em39me7-750 & 15.6 & $\star * \star * *$ \\
\hline NV 2012 & Dnv2-1 & LG1 & 58.9 & 3.4 & em1me26-255 & 11.3 & - \\
\hline SLW 2011 & Ds/w1-1 & LG22 & 35.5 & 3.3 & em5me14-270 & 25.9 & - \\
\hline \multirow[t]{4}{*}{ SLW 2012} & Ds/w2-1 & LG1 & 106.8 & $7.3^{\star \star}$ & em4me11-140c & 29.6 & $\star \star \star \star \star \star \star * *$ \\
\hline & Ds/w2-2 & LG7 & 67.0 & $4.9^{* *}$ & em7me28-200 & 16.5 & $\star \star * * * * * *$ \\
\hline & Ds/w2-3 & LG9 & 45.2 & 3.5 & DZ132-260 & 19.5 & - \\
\hline & Ds/w2-4 & LG22 & 13.0 & 3.3 & em15me23-360 & 44.5 & - \\
\hline \multirow[t]{3}{*}{ NL 2012} & $D n / 2-1$ & LG2 & 104.0 & 3.8 & E6M4-130 & 24.9 & - \\
\hline & $D n / 2-2$ & LG10 & 52.2 & 4.1 & em39me7-1500 & 51.2 & $\star *$ \\
\hline & Dnl2-3 & LG18 & 68.6 & 3.1 & em3me14-700 & 38.4 & - \\
\hline TDW 2011 & Dtdw1-1 & LG10 & 35.0 & 3.0 & em52me12-340 & 29.9 & - \\
\hline \multirow[t]{3}{*}{ TDW 2012} & Dtdw2-1 & LG1 & 104.8 & 3.9 & em4me11-140c & 11.2 & 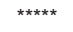 \\
\hline & Dtdw2-2 & LG2 & 104.0 & $6.2^{* *}$ & E6M4-130 & 38.0 & - \\
\hline & $D t d w 2-3$ & LG18 & 0.0 & $4.7^{* *}$ & em3me14-820c & 36.4 & - \\
\hline \multirow[t]{5}{*}{ CA 2011} & Dca1-1 & LG1 & 97.7 & $7.3^{\star *}$ & em13me4-290c & 15.1 & 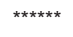 \\
\hline & Dca1-2 & LG1 & 129.0 & $7.3^{\star *}$ & em1 me14-2400c & 19.7 & 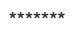 \\
\hline & Dca1-3 & LG9 & 68.6 & $4.6^{\star \star}$ & em9me9-190 & 20.2 & - \\
\hline & Dca1-4 & LG10 & 15.9 & 3.3 & em31me26-160 & 12.1 & $* * * *$ \\
\hline & Dca1-5 & LG14 & 32.5 & $4.7^{\text {** }}$ & em3me2-655 & 32.1 & - \\
\hline \multirow[t]{3}{*}{ CA 2012} & Dca2-1 & LG1 & 35.0 & $5.6^{\star *}$ & em56me4-50 & 19.1 & $* * * * *$ \\
\hline & Dca2-2 & LG1 & 129.0 & $9.5^{\star \star}$ & em1 1 me14-2400c & 27.8 & 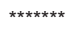 \\
\hline & Dca2-3 & LG7 & 39.3 & 3.3 & em7me28-240 & 21.2 & ** \\
\hline
\end{tabular}




\begin{tabular}{|c|c|c|c|c|c|c|c|}
\hline Traits $^{a}$ & $\mathrm{QTL}^{b}$ & Linkage group & Position $(\mathrm{cM})^{c}$ & $\mathrm{LOD}^{d}$ & Marker ${ }^{e}$ & $\%$ Var. $^{f}$ & $\mathrm{KW}^{g}$ \\
\hline & Dca2-3 & LG7 & 39.3 & 3.3 & em7me28-240 & 21.2 & ** \\
\hline & Dca2-4 & LG18 & 44.6 & $4.9^{* \star}$ & em3me14-2400 & 49.6 & - \\
\hline & Dca2-5 & LG18 & 106.5 & 3.8 & em11me14-300c & 10.4 & **** \\
\hline & Dca2-6 & LG22 & 16.6 & 3.2 & em15me23-360 & 53.3 & * \\
\hline \multirow[t]{3}{*}{ RU 2011} & Dru1-1 & LG1 & 92.8 & $5.0^{\star \star}$ & em4me7-390 & 39.6 & - \\
\hline & Dru1-2 & LG7 & 22.7 & 3.0 & em44me26-190 & 37.5 & - \\
\hline & Dru1-3 & LG9 & 73.8 & 3.2 & em9me9-170 & 12.4 & $* * *$ \\
\hline \multirow[t]{6}{*}{ RU 2012} & Dru2-1 & LG1 & 41.4 & $4.2^{* *}$ & em4me3-2350 & 13.8 & $\star * * * * * * *$ \\
\hline & Dru2-2 & LG1 & 129.0 & $4.5^{\star \star}$ & em1me14-2400c & 14.3 & 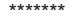 \\
\hline & Dru2-3 & LG7 & 40.3 & $4.5^{\star \star}$ & em7me28-240 & 20.1 & $\star * * * *$ \\
\hline & Dru2-4 & LG7 & 59.0 & 3.8 & em9me13-170 & 40.4 & - \\
\hline & Dru2-5 & LG9 & 88.1 & 3.9 & em13me4-180c & 24.6 & ** \\
\hline & Dru2-6 & LG20 & 14.2 & 4.0 & em4me13-1620 & 39.3 & - \\
\hline \multirow[t]{3}{*}{ EUR 2011} & Deur1-1 & LG1 & 71.0 & $5.0^{\star \star}$ & em49me3-150 & 14.5 & 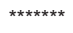 \\
\hline & Deur1-2 & LG1 & 117.5 & $5.2^{\star \star}$ & DZ159-161c & 14.6 & $* \star * * * * * *$ \\
\hline & Deur1-3 & LG2 & 114.9 & 3.1 & E5M4-500 & 12.2 & $* *$ \\
\hline EUR 2012 & Deur2-1 & LG1 & 70.9 & $7.6^{\star \star}$ & em1me26-2400 & 21.4 & - \\
\hline
\end{tabular}

${ }^{a}$ Abbreviations of traits are the same as those listed in Table 2. ${ }^{b} \mathrm{QTL}$ was named using an abbreviation of the trait followed by the year ( 1 for 2011 and 2 for 2012) and the QTL number. 'Position: logarithm of odds (LOD) peak position. वLOD: maximum LOD value, ${ }^{* *}$ LOD value significant at $\mathrm{P}<0.05$ based on 1000 genome-wide permutation tests. ${ }^{e} \mathrm{Marker:}$ marker name nearest to the QTL position. ${ }^{f} \%$ Var.: proportion of the total phenotypic variance explained by the QTL. ${ }^{g} \mathrm{KW}$ : Kruskal-Wallis significance level, given by the P values $\left({ }^{*} 0.1,{ }^{* *} 0.05,{ }^{* * *} 0.01,{ }^{* * * *} 0.005,{ }^{* * * * *} 0.001,{ }^{* * * * * *} 0.0005\right.$, $* * * * * * * 0.0001)$.

Fifteen QTL clusters that showed significant marker phenotype associations with at least two leaf traits were found on LG1, LG2, LG5, LG7, LG9, LG10, LG14, LG18, and LG22 (Figure 2). Overlapping confidence intervals between chlorogenic acid QTLs and rutin QTLs were found on LG1 (Dca1-2, Dca2-2, and Dru2-2), LG7 (Dca2-3 and Dru2-3), and LG9 (Dca1-3, Dru1-3, and Dru2-5). Six QTL regions on LG1, LG2, LG7, LG10, LG18, and LG22, which affected secondary metabolites, were also found to affect, by extension, leaf morphological characters or yield-related traits. The other six QTL clusters influenced at least three of the leaf morphological characters or yield-related traits. Sixteen QTLs were unique to specific traits.

\section{DISCUSSION}

There is promising potential to exploit and utilize E. ulmoides, but genetic improvement of economically important traits is a challenging task for breeders. Genetic analyses of the complex quantitative traits involved in leaf morphology, yield, and secondary metabolites are limited. This preliminary study aimed to understand the genetic architecture of these economically important traits.

The estimated magnitude of the individual QTL effects ranged from 10.4 to $53.3 \%$ of the phenotypic variance (Table 3). Similarly, we were able to detect QTLs with a moderate-to-large effect for plant height and diameter growth in the same mapping population (Li et al., 2014c). A few QTLs with relatively large effect have also been observed for growth, phenology, quality, and yield traits in other plant species (Bradshaw and Stettler, 1995; Wang et al., 2000; Ronnberg-Wastljung et al., 2005; McAdam et al., 2013). This indicates that these quantitative traits may be controlled by a few genes that have a large effect. Nevertheless, the limited population size used in the present QTL study could lead to overestimation of QTL effects and underestimation of QTL number (Beavis, 1998). Bradshaw et al. $(1995,1998)$ reported QTL detection for floral morphology traits in monkeyflower using mapping populations of 93 and $465 F_{2}$ individuals. For the 11 QTLs common 
to the two populations, the size of the QTL effects was reduced in the larger population, suggesting an upward bias of magnitude estimates in small mapping populations. In barley, Vales et al. (2005) found that QTLs with a large effect could be detected even in small populations, whereas those with a small effect could only be detected by increasing the population size.

Co-location of the putative QTLs identified for different leaf traits was a striking feature of this study. Fifteen distinct QTL clusters, each harboring loci controlling at least two leaf traits, were detected across nine linkage groups in the DZ0901 population (Figure 2). Of these 15 QTL regions, 12 were found to affect at least three of the leaf morphological characteristics or yield-related traits. This was not surprising because of the strong phenotypic correlations among these traits (Table 2). The remaining three QTL regions detected on LG1, LG7, and LG9 were found to affect chlorogenic acid and rutin (Figure 2). Furthermore, highly significant positive correlations were found between chlorogenic acid and rutin (Table 2). Previous QTL studies on main crops have frequently detected regions of the genome that contain clusters of QTLs for highly correlative traits (Zeng et al., 2009; Avia et al., 2013; Bian et al., 2013). Clusters of QTLs were also observed for growth traits and various leaf traits in poplar (Bradshaw and Stettler, 1995; Wu et al., 1997), for wood properties and growth traits in the genus Eucalyptus (Verhaegen et al., 1997; Freeman et al., 2009; Thumma et al., 2010), for growth traits in Salix (Tsarouhas et al., 2002; Ronnberg-Wastljung et al., 2005), for leaf morphological characters in European beech (Scalfi et al., 2004) and oak (Gailing, 2008; Gailing et al., 2013), and for chemical compositions in peach (Eduardo et al., 2013) and oil palm (Montoya et al., 2013). Furthermore, the clustering of QTLs controlling height and basal diameter has been observed in the same mapping population of E. ulmoides (Li et al., 2014c). In most cases, no clear patterns among phenotypic correlations have been found to coincide with the co-locating QTLs (Tsarouhas et al., 2002; Gailing, 2008; Freeman et al., 2009; Thumma et al., 2010; Li et al., 2014c). In the present study, we found that 16 QTLs were unique to specific traits (Figure 2).

Co-location of QTLs for multiple traits may result from the pleiotropic effect of major genes in these genomic regions (McAdam et al., 2013; Zhang et al., 2013). It is possible that a single gene could independently control either two or more different traits. Alternatively, these genomic locations may contain several genes, each of which affects a different trait. Pleiotropy is consistent with the conclusion that chlorogenic acid and rutin are derived from common precursors and pathways of phenylpropanoid biosynthesis (Zhang et al., 2002). We detected 12 QTL regions, which each affected at least three of the leaf traits in the DZ0901 mapping population. Compared with our previous QTL study in the same population, the QTL regions on LG10, LG18, and LG22 were also found to affect height and diameter growth ( $\mathrm{Li}$ et al., 2014c). These genomic regions may contain major genes that control the growth of the whole plant in E. ulmoides. In addition, the co-location of these QTLs may be due to the linkage of loci that are associated with different traits.

We focused on increasing the secondary metabolite content and leaf yield to enhance the final secondary metabolite yield. In this study, six QTL regions that affected secondary metabolites were also found to affect at least three of the leaf morphological characteristics or yield-related traits (Figure 2). In breeding programs, special attention should be given to these QTL regions, which may be useful in the simultaneous improvement of secondary metabolite content and leaf yield. However, use of these QTLs in molecular E. ulmoides breeding may have undesirable consequences. When the same QTL affects different and opposing traits, MAS is unlikely to succeed (Heslop-Harrison and Schwarzacher, 2011). Furthermore, low phenotypic correlations between secondary metabolites and other leaf traits were estimated in the DZ0901 population. This issue should be verified in other mapping population of E. ulmoides.

The low correlations observed for leaf traits between years may be due to the highly 
divergent environmental conditions in the field in 2011 and 2012. For the six leaf morphological characteristics, no QTL was consistently detected over the two study years. The same observations were made for the number of leaves and the total dry weight. The instability of QTLs over years has been reported for leaf traits in other tree species (Scalfi et al., 2004; Gailing, 2008; Gailing et al., 2013). Fanizza et al. (2005) hypothesized that QTL instability over time may be due to the presence of different genes or the differential expression of these genes due to changes in genotypic sensitivity to annual variations in the climatic conditions. Plomion et al. (1996) assumed that maturation in trees induces a progressive shift in the genetic control of phenotypic traits. This may provide a possible explanation for the low year-to-year correlation between leaf traits.

Three QTL regions for secondary metabolites were consistently identified in each year (Figure 2, Table 3). The peak LOD value for the chlorogenic acid QTLs, Dca1-2 and Dca2-2, was at $129.0 \mathrm{cM}$ on LG1, which was tightly linked with the marker em1me14-2400c. QTL Dca1-2, identified in 2011 with an LOD score of 7.3, was estimated to explain $19.7 \%$ of the phenotypic variance. QTL Dca2-2, identified in 2012 with an LOD score of 9.5, was found to explain $27.8 \%$ of the phenotypic variance. A rutin QTL Dru2-2, identified in 2012, had the same peak position as Dca1-2 and Dca2-2; thus, these three might be the same QTL. The two QTLs for rutin, Dru1-3 in 2011 and Dru2-5 in 2012, were located in a similar region of LG9, and were co-located with a QTL for chlorogenic acid (Dca1-3). For EU-rubber, a stable QTL region over years was identified on LG1 (Deur1-1 in 2011 and Deur2-1 in 2012). Our results are specific to the DZ0901 mapping population. For practical MAS, the QTL information should be confirmed in different populations across different locations and years.

\section{Conflicts of interest}

The authors declare no conflict of interest.

\section{ACKNOWLEDGMENTS}

Research supported by the National Natural Science Foundation of China (\#30771751).

\section{REFERENCES}

Avia K, Pilet-Nayel ML, Bahrman N, Baranger A, et al. (2013). Genetic variability and QTL mapping of freezing tolerance and related traits in Medicago truncatula. Theor. Appl. Genet. 126: 2353-2366.

Beavis WB (1998). QTL Analyses: Power, Precision, and Accuracy. In: Molecular Dissection of Complex Traits (Paterson AH, eds.). CRC Press, Boca Raton, 145-162.

Bian JM, Shi H, Li CJ, Zhu CL, et al. (2013). QTL mapping and correlation analysis for 1000-grain weight and percentage of grains with chalkiness in rice. J. Genet. 92: 281-287.

Bradshaw HD and Stettler RF (1995). Molecular genetics of growth and development in Popolus. IV. Mapping QTLs with large effects on growth, form and phenology traits in a forest tree. Genetics 139: 963-973.

Bradshaw HD, Wilbert SM, Otto KG and Schemske DW (1995). Genetic mapping of floral traits associated with reproductive isolation in monkeyflowers (Mimulus). Nature 376: 762-765.

Bradshaw HD, Otto KG, Frewen BE, McKay JK, et al. (1998). Quantitative trait loci affecting differences in floral morphology between two species of monkeyflower (Mimulus). Genetics 149: 367-382.

China Pharmacopoeia Committee (2005). Chinese pharmacopoeia (volume 1). 8th edn. Chemical Industry Press, Beijing.

Eduardo I, Chietera G, Pirona R, Pacheco I, et al. (2013). Genetic dissection of aroma volatile compounds from the essential oil of peach fruit: QTL analysis and identification of candidate genes using dense SNP maps. Tree Genet. Genom. 9: 189-204.

Fanizza G, Lamaj F, Costantini L, Chaabane R, et al. (2005). QTL analysis for fruit yield components in table grapes (Vitis vinifera). Theor. Appl. Genet. 111: 658-664. 
Freeman JS, Whittock SP, Potts BM and Vaillancourt RE (2009). QTL influencing growth and wood properties in Eucalyptus globulus. Tree Genet. Genom. 5: 713-722.

Gailing O (2008). QTL analysis of leaf morphological characters in a Quercus robur full-sib family (Q. robur x Q. robur ssp. slavonica). Plant Biol. 10: 624-634.

Gailing O, Bodénès C, Finkeldey R, Kremer A, et al. (2013). Genetic mapping of EST-derived simple sequence repeats (ESTSSRs) to identify QTL for leaf morphological characters in a Quercus robur full-sib family. Tree Genet. Genom. 9: 1361-1367.

Heslop-Harrison JS and Schwarzacher T (2011). Genetics and Genomics of Crop Domestication. In: Plant Biotechnology and Agriculture: Prospects for the 21st Century ( Altman A and Hasegawa PM, eds.). Academic Press, London, 1-16.

Hsieh CL and Yen GC (2000). Antioxidant actions of Du-zhong (Eucommia ulmoides Oliv.) toward oxidative damage in biomolecules. Life Sci. 66: 1387-1400.

Hu SY (1979). A contribution to our knowledge of tu-chung - Eucommia ulmoides. Am. J. Chin. Med. 7: 5-37.

Kwan CY, Chen CX, Deyama T and Nishibe S (2003). Endothelium-dependent vasorelaxant effects of the aqueous extracts of the Eucommia ulmoides Oliv. leaf and bark: implications on their antihypertensive action. Vascul. Pharmacol. 40: 229-235.

Lee MK, Kim MJ, Cho SY, Park SA, et al. (2005). Hypoglycemic effect of Du-zhong (Eucommia ulmoides Oliv.) leaves in streptozotocin-induced diabetic rats. Diabetes Res. Clin. Pract. 67: 22-28.

Li Y, Wang SH, Li ZQ, Jin CF, et al. (2014a). Genetic diversity and relationships among Chinese Eucommia ulmoides cultivars revealed by sequence-related amplified polymorphism, amplified fragment length polymorphism, and inter-simple sequence repeat markers. Genet. Mol. Res. 13: 8704-8713.

Li Y, Wei YC, Li ZQ, Wang SH, et al. (2014b). Relationship between progeny growth performance and molecular marker-based genetic distances in Eucommia ulmoides parental genotypes. Genet. Mol. Res. 13: 4736-4746.

Li Y, Wang DW, Li ZQ, Wei JK, et al. (2014c). A molecular genetic linkage map of Eucommia ulmoides and quantitative trait loci (QTL) analysis for growth traits. Int. J. Mol. Sci. 15: 2053-2074.

Ma BL, Wang L, Zhang KJ and Zhang YX (1994). Study on extraction method of Eucommia ulmoides rubber in laboratory. J. NW Forest. Univ. 9: 67-69.

McAdam EL, Freeman JS, Whittock SP, Buck EJ, et al. (2013). Quantitative trait loci in hop (Humulus lupulus L.) reveal complex genetic architecture underlying variation in sex, yield and cone chemistry. BMC Genomics 14: 360.

Montoya C, Lopes R, Flori A, Cros D, et al. (2013). Quantitative trait loci (QTLs) analysis of palm oil fatty acid composition in an interspecific pseudo-backcross from Elaeis oleifera (H.B.K.) Cortes and oil palm (Elaeis guineensis Jacq.). Tree Genet. Genom. 9: 1207-1225.

Nakamura T, Nakazawa Y, Onizuka S, Satoh S, et al. (1997). Antimutagenicity of Tochu tea (an aqueous extract of Eucommia ulmoides leaves): 1. The clastogen-suppressing effects of Tochu tea in $\mathrm{CHO}$ cells and mice. Mutat. Res. 388: 7-20.

Nakazawa Y, Bamba T, Takeda T, Uefuji H, et al. (2009). Production of Eucommia-rubber from Eucommia ulmoides Oliv. (Hardy Rubber Tree). Plant Biotechnol. 26: 71-79.

Plomion C, Durel CE and O'Malley DM (1996). Genetic dissection of height in maritime pine seedlings raised under accelerated growth conditions. Theor. Appl. Genet. 93: 849-858.

Rönnberg-Wästljung AC, Glynn C and Weih M (2005). QTL analyses of drought tolerance and growth for a Salix dasyclados x Salix viminalis hybrid in contrasting water regimes. Theor. Appl. Genet. 110: 537-549.

Scalfi M, Troggio M, Piovani P, Leonardi S, et al. (2004). A RAPD, AFLP and SSR linkage map, and QTL analysis in European beech (Fagus sylvatica L.). Theor. Appl. Genet. 108: 433-441.

Takamura C, Hirata T, Yamaguchi Y, Ono M, et al. (2007). Studies on the chemical constituents of green leaves of Eucommia ulmoides Oliv. J. Nat. Med. 61: 220-221.

Thumma BR, Baltunis BS, Bell JC, Emebiri LC, et al. (2010). Quantitative trait locus (QTL) analysis of growth and vegetative propagation traits in Eucalyptus nitens full-sib families. Tree Genet. Genom. 6: 877-889.

Tsarouhas V, Gullberg U and Lagercrantz U (2002). An AFLP and RFLP linkage map and quantitative trait locus (QTL) analysis of growth traits in Salix. Theor. Appl. Genet. 105: 277-288.

Vales MI, Schön CC, Capettini F, Chen XM, et al. (2005). Effect of population size on the estimation of QTL: a test using resistance to barley stripe rust. Theor. Appl. Genet. 111: 1260-1270.

Van Ooijen JW (2004). MapQTL 5, Software for the mapping of quantitative trait loci in experimental populations. Kyazma B.V., Wageningen.

Verhaegen D, Plomion C, Gion JM, Poitel M, et al. (1997). Quantitative trait dissection analysis in Eucalyptus using RAPD markers. 1. Detection of QTL in interspecific hybrid progeny, stability of QTL expression across different ages. Theor. Appl. Genet. 95: 597-608.

Voorrips RE (2002). MapChart: Software for the graphical presentation of linkage maps and QTLs. J. Hered. 93: 77-78.

Wang D, Karle R and lezzoni AF (2000). QTL analysis of flower and fruit traits in sour cherry. Theor. Appl. Genet. 100: 535-544.

Wu R, Bradshaw HD and Stettler RF (1997). Molecular genetics of growth and development in Populus (Salicaceae). V. Mapping quantitative trait loci affecting leaf variation. Am. J. Bot. 84: 143-153.

Genetics and Molecular Research 14 (4): 17871-17884 (2015) ～CFUNPEC-RP www.funpecrp.com.br 
Zeng GL, Li DM, Han YP, Teng WL, et al. (2009). Identification of QTL underlying isoflavone contents in soybean seeds among multiple environments. Theor. Appl. Genet. 118: 1455-1463.

Zhang KJ, Wang L and Ma BL (2002). Chlorogenic Acid in Eucommia ulmoides. In: Secondary Metabolites in Chinese Eucommia Ulmoides. Science Press, Beijing, 171-180.

Zhang R, Wu J, Li XG, Khan MA, et al. (2013). An AFLP, SRAP, and SSR genetic linkage map and identification of QTLs for fruit traits in pear (Pyrus L.). Plant Mol. Biol. Rep. 31: 678-687. 